\title{
Avaliação dos Desempenhos de Estimadores para os Parâmetros da Distribuição Birnbaum-Saunders
}

\author{
D.B.M. MACIEL, G.I.A. VIEIRA* e P.R.D. MARINHO
}

Recebido em 2 agosto, 2012 / Aceito em 6 dezembro, 2013

\begin{abstract}
RESUMO. Este artigo teve como objetivo principal avaliar os desempenhos dos estimadores dos Métodos dos Momentos Modificados para os parâmetros $\alpha$ e $\beta$ da distribuição Birnbaum-Saunders e três versões corrigidas desses estimadores: a versão corrigida por viés, a corrigida por viés via jackknife e a versão corrigida por viés via bootstrap. Simulações de Monte Carlo foram utilizadas para a realização do objetivo proposto através da verificação de algumas propriedades desses estimadores, a saber: média e erro.
\end{abstract}

Palavras-chave: distribuição Birnbaum-Saunders, correção de viés, estimativas jackknife e bootstrap.

\section{INTRODUÇÃO}

A distribuição Birnbaum-Saunders biparamértica [2] de parâmetros $\alpha$ e $\beta$ vem sendo amplamente usada em ciências da engenharia para modelar o tempo de vida de materiais e equipamentos. $\mathrm{Na}$ prática, os parâmetros que indexam a distribuição são estimados a partir de dados coletados. Os estimadores usuais, contudo, podem não apresentar desempenho satisfatório em um cenário de tamanho amostral pequeno. Logo, torna-se necessária a obtenção de estimadores que sejam menos tendenciosos em amostras pequenas.

No caso da distribuição Birnbaum-Saunders, tem-se uma outra problemática: os estimadores do método dos momentos para $\alpha$ e $\beta$ não existem caso o coeficiente de variação amostral seja maior do que $\sqrt{5}$ e existem caso essa estatística seja inferior a $\sqrt{5}$, contudo, o estimador de $\beta$ não é único. Baseado nisso, [8] propôs um estimador alternativo: o estimador do método dos momentos modificados (EMMMs), os quais não sofrem dos problema apresentados pelos estimadores de momentos tradicionais de $\alpha$ e $\beta$.

Este artigo tem como objetivo avaliar os desempenhos dos EMMMs e suas versões corrigidas através de simulações de Monte Carlo. Foram consideradas as correções por viés obtidas em [8], as correções por viés via jackknife, propostas por [5, 6], além de ser apresentada uma versão corrigida por viés via bootstrap.

*Autor correspondente: Giannini Italino Alves Vieira

Mestrando do Programa de Pós-Graduação em Estatística, PPGE-UFPE.

E-mails:dbm.maciel@gmail.com; italino.ufpe@gmail.com; pedro.rafael.marinho@gmail.com 
Após esta seção introdutória, segue uma breve discussão sobre as principais características da distribuição Birnbaum-Saunders. Na Seção 3, apresenta-se e discute-se, brevemente, cada estimador proposto. Na Seção 4 constam os resultados e discussões. Em seguida, o artigo é finalizado com as principais conclusões encontradas.

\section{A DISTRIBUIÇÃO BIRNBAUM-SAUNDERS}

A distribuição Birnbaum-Saunders $(\mathcal{B} S)$ teve como origem o desenvolvimento de uma família de distribuições para modelar o tempo de vida de materiais e equipamentos sujeitos a cargas dinâmicas que os levam à ocorrência de falhas. Estas falhas acontecem como resultado do desenvolvimento e crescimento de uma fissura dominante em materiais sujeitos a um padrão cíclico de pressão. Denomina-se por fadiga de um material a falha estrutural que resulta quando este é submetido a diferentes esforços dinâmicos repetidos ou oscilações dinâmicas.

Na prática, os modelos estatísticos para processos de fadiga de material produzem a descrição do tempo de falha até a ocorrência da fadiga de um determinado material ou equipamento, tempo este não necessariamente constante para materiais sob condições fatigantes.

Para entender o problema que levou à proposição da distribuição Birnbaum-Saunders, assuma que, para um certo equipamento dentro das condições acima mencionadas, o $i$-ésimo ciclo gera um crescimento aleatório na fissura de dimensão $X_{i}$, com a extensão da mesma até o $m$-ésimo $\operatorname{ciclo}^{1}\left(Y_{m}\right)$ dada por

$$
Y_{m}=\sum_{i=1}^{m} X_{i}
$$

em que $Y_{m}$ é uma variável aleatória com média $m \mu$ e variância $m \sigma^{2}$, para todo $m=1,2,3 \ldots$, com $m$ representando o número de ciclos.

Além disso, a probabilidade que a fissura não ultrapasse uma certa extensão crítica $w$, após $m$ ciclos, é

$$
H_{m}(w)=\mathbb{P}\left(Y_{m} \leq w\right)
$$

para $m=1,2,3 \ldots$

Se $C$ representa o número de ciclos até a falha, que esta ocorrendo quando o comprimento da fissura excede um certo nível crítico $w$, então têm-se

$$
\mathbb{P}(C \leq m)=\mathbb{P}\left(\sum_{i=1}^{m} X_{i} \geq w\right)=1-H_{m}(w) .
$$

Nota-se que a função de distribuição de $C$ pode ser aproximada utilizando o Teorema do Limite Central, bastando assumir que as variáveis aleatórias $X_{i}$ são independentes e identicamente distribuídas (no caso, com média $\mu$ e variância $\sigma^{2}$ ). Então tem-se:

\footnotetext{
${ }^{1}$ Assume-se aqui, para efeito de simplificação, que um ciclo é composto por uma única oscilação.
} 


$$
\begin{aligned}
\mathbb{P}(C \leq m) & =1-\mathbb{P}\left(\sum_{i=1}^{m} \frac{X_{i}-\mu}{\sigma \sqrt{m}} \leq \frac{w}{\sigma \sqrt{m}}-\frac{\mu \sqrt{m}}{\sigma}\right) \\
& \approx 1-\Phi\left(\frac{w}{\sigma \sqrt{m}}-\frac{\mu \sqrt{m}}{\sigma}\right)=\Phi\left(\frac{\mu \sqrt{m}}{\sigma}-\frac{w}{\sigma \sqrt{m}}\right),
\end{aligned}
$$

em que $\Phi$ representa a função distribuição acumulada normal padrão.

Se $m$ é substituído por uma variável real não negativa $t$, têm-se que a variável aleatória $T$ pode ser vista como a extensão contínua da variável aleatória discreta $C$, o que torna $T$ o tempo até a falha.

Então, a função de distribuição acumulada para esta variável pode ser escrita como

$$
\begin{aligned}
\mathbb{P}(C \leq m) \equiv \mathbb{P}(T \leq t) & =\Phi\left[\frac{\mu \sqrt{t}}{\sigma}-\frac{w}{\sigma \sqrt{t}}\right]=\Phi\left[\frac{\sqrt{w} \sqrt{\mu}}{\sigma}\left(\frac{\sqrt{\mu} \sqrt{t}}{\sqrt{w}}-\frac{\sqrt{w}}{\sqrt{t} \sqrt{\mu}}\right)\right] \\
& =\Phi\left[\frac{1}{\alpha}\left(\sqrt{\frac{t}{\beta}}-\sqrt{\frac{\beta}{t}}\right)\right],
\end{aligned}
$$

em que $\alpha=\frac{\sigma}{\sqrt{w \mu}}>0, \beta=\frac{w}{\mu}>0$ e $t>0$.

Neste caso, diz-se que a variável aleatória $T$ segue distribuição Birnbaum-Saunders biparamétrica, ou seja $T \sim \mathcal{B S}(\alpha, \beta)$, com $\alpha$ como parâmetro de forma e $\beta$ como parâmetro de escala. Note que $\beta$ é a mediana da distribuição $\mathcal{B} S$.

A função densidade de probabilidade de $T$ é dada por:

$$
\begin{aligned}
f_{T}(t, \alpha, \beta) & =\phi\left(\frac{1}{\alpha}\left[\sqrt{\frac{t}{\beta}}-\sqrt{\frac{\beta}{t}}\right]\right) \times \frac{d}{d t}\left\{\frac{1}{\alpha}\left[\sqrt{\frac{t}{\beta}}-\sqrt{\frac{\beta}{t}}\right]\right\} \\
& =\frac{1}{2 \alpha \beta \sqrt{2 \pi}}\left[\left(\frac{\beta}{t}\right)^{1 / 2}+\left(\frac{\beta}{t}\right)^{3 / 2}\right] \exp \left\{-\frac{1}{2 \alpha^{2}}\left(\frac{t}{\beta}+\frac{\beta}{t}-2\right)\right\},
\end{aligned}
$$

em que $\phi$ é a densidade normal padrão, com $\alpha>0, \beta>0$ e $t>0$. A média, variância, assimetria e curtose da distribuição Birnbaum-Saunders são dadas respectivamente por

$$
\begin{aligned}
\mathbb{E}(T) & =\beta\left(1+\frac{\alpha^{2}}{2}\right) \\
\operatorname{Var}(T) & =(\alpha \beta)^{2}\left(1+\frac{5}{4} \alpha^{2}\right), \\
\mu_{3} & =\frac{4 \alpha\left(11 \alpha^{2}+6\right)}{\left(5 \alpha^{2}+4\right)^{3 / 2}} \\
\mu_{4} & =3+\frac{6 \alpha^{2}\left(93 \alpha^{2}+40\right)}{\left(5 \alpha^{2}+4\right)^{2}},
\end{aligned}
$$

com as expressões para assimetria $\left(\mu_{3}\right)$ e curtose $\left(\mu_{4}\right)$ de acordo com a correção proposta por [6]. 
Seja $T$ uma variável aleatória contínua com distribuição $\mathcal{B} S(\alpha, \beta)$. A distribuição BirnbaumSaunders possui algumas propriedades interessantes $[1,3,7]$ :

1. $\alpha$ é um parâmetro de forma, tal que, quando $\alpha \rightarrow 0$, a distribuição $\mathcal{B} S$ tende para uma distribuição normal biparamétrica $N(\beta, \tau)(\operatorname{com} \tau \rightarrow 0$ quando $\alpha \rightarrow 0)$;

2. $\beta$ é um parâmetro de escala, ou seja $T / \beta \sim \mathcal{B S}(\alpha, 1)$;

3. Para qualquer constante real, $k>0$, têm-se $k T \sim \mathcal{B} S(\alpha, k \beta)$;

4. A distribuição $\mathcal{B S}$ é recíproca, ou seja, se $T \sim \mathcal{B S}(\alpha, \beta)$, então $T^{-1} \sim \mathcal{B} S\left(\alpha, \beta^{-1}\right)$, que pertence à mesma família de distribuições da $\mathcal{B} S$.

\section{PROCEDIMENTOS DE INFERÊNCIA}

Um dos principais problemas da inferência estatística é o da estimação. Tem-se tal problema quando, por exemplo, deseja-se obter um estimador para os parâmetros $\alpha$ e $\beta$ de $\mathcal{B} S(\alpha, \beta)$. Esse estimadores deverão ser consistentes e de preferência com o menor viés possível. Uma forma adequada e tipicamente utilizada para comparar diferentes estimadores é utilizar o erro quadrático médico.

\subsection{Estimador do Método dos Momentos Modificados $\tilde{\alpha}$ e $\tilde{\beta}$}

O método dos momentos é uma metodologia de estimação que consiste em igualar os momentos populacionais aos respectivos momentos amostrais e resolver o sistema de equações resultante. Porém, conforme mencionado na Seção 1, no caso da distribuição Birnbaum-Saunders, existem dificuldades para obtenção desses estimadores. Diante disso, [8] propôs o estimador do método dos momentos modificados. A ideia consiste em utilizar os momentos populacionais apresentados abaixo.

$$
\begin{aligned}
\mathbb{E}(T) & =\beta\left(1+\frac{1}{2} \alpha^{2}\right), \\
\mathbb{E}\left(T^{-1}\right) & =\beta^{-1}\left(1+\frac{1}{2} \alpha^{2}\right) .
\end{aligned}
$$

Igualando-se os momentos amostrais com os respectivos momentos populacionais apresentados logo acima, obtém-se

$$
\begin{aligned}
s & =\beta\left(1+\frac{1}{2} \alpha^{2}\right), \\
r^{-1} & =\beta^{-1}\left(1+\frac{1}{2} \alpha^{2}\right),
\end{aligned}
$$

em que $s=\frac{1}{n} \sum_{i=1}^{n} t_{i}, r=\left[\frac{1}{n} \sum_{i=1}^{n} t_{i}^{-1}\right]$ e $t_{i}$ são valores de uma amostra aleatória de tamanho $n$. Note que $s$ e $r$ são, respectivamente, a média aritmética e a média harmônica de $t_{i}, i=$ $1, \ldots, n$. 
Resolvendo as equações (3.1) e (3.2) em relação a $\alpha$ e $\beta$ obtém-se os EMMMs, denotados por $\widetilde{\alpha}$ e $\widetilde{\beta}$, em que

$$
\widetilde{\alpha}=\left\{2\left[\left(\frac{s}{r}\right)^{\frac{1}{2}}-1\right]\right\}^{\frac{1}{2}} \text { e } \quad \widetilde{\beta}=(s r)^{\frac{1}{2}} .
$$

Os estimadores obtidos pelo método dos momentos são, em geral, consistentes e possuem distribuição assintótica gaussiana, porém não são os mais eficientes assintoticamente.

\subsection{Estimador do Método dos Momentos Modificados, corrigido por viés $\check{\alpha}$ e $\check{\beta}$}

[6] notou que os vieses de $\widetilde{\alpha}$ e $\widetilde{\beta}$, podem ser aproximados por, respectivamente,

$$
\operatorname{viés}(\widetilde{\alpha}) \approx-\frac{\alpha}{n}, \operatorname{viés}(\widetilde{\beta}) \approx \frac{\alpha^{2}}{4 n} \text {. }
$$

Consequentemente, a partir da definição de viés de um estimador , tem-se que

$$
\begin{aligned}
\mathbb{E}(\tilde{\alpha}) \approx \alpha-\frac{\alpha}{n} & =\left(\frac{n-1}{n}\right) \alpha, \\
\mathbb{E}(\tilde{\beta}) \approx \beta+\frac{\alpha^{2} \beta}{4 n} & =\left(1+\frac{\alpha^{2}}{4 n}\right) \beta,
\end{aligned}
$$

o que leva aos estimadores de $\alpha$ e $\beta$ corrigidos por viés propostos [8],

$$
\check{\alpha}=\left(\frac{n}{n-1}\right) \tilde{\alpha} \quad \text { e } \check{\beta}=\left(1+\frac{\tilde{\alpha}^{2}}{4 n}\right)^{-1} \tilde{\beta} .
$$

Em [5,6] menciona-se que as expressões dos vieses encontradas por [6] não possuem embasamento teórico, uma vez que foram obtidas após um longo estudo de simulação de Monte Carlo, com posterior análise descritiva e visual dos resultados.

\subsection{Estimador do Método dos Momentos Modificados, corrigido por viés via Jackknife $\bar{\alpha}$ e $\bar{\beta}$}

A ideia do método jackknife é remover a observação $t_{j}$ da amostra aleatória $T_{n}=\left\{t_{1}, t_{2}, \ldots t_{n}\right\}$ (amostra original) e estimar os parâmetros baseados nas $n-1$ observações restantes; isto é feito para $j=1, \ldots, n$. Toda quantidade subscrita por $(j)$ refere-se a uma quantidade calculada na amostra de tamanho $n-1$, ou seja, na amostra sem a observação $t_{j}$. Suponha-se que uma obervação $j$ é retirada. Então, baseado em [5,6] teríamos

$$
\begin{aligned}
s_{(j)} & =\frac{1}{n-1} \sum_{\substack{i=1 \\
i \neq j}}^{n} t_{i}=\frac{n s-t_{j}}{n-1}, \\
r_{(j)} & =\left[\frac{1}{n-1} \sum_{\substack{i=1 \\
i \neq j}}^{n} t_{j}^{-1}\right]^{-1}=\frac{n r-t_{j}^{-1}}{n-1} .
\end{aligned}
$$


Desta forma, temos que

$$
\begin{aligned}
& \tilde{\alpha}_{(j)}=\left\{2\left[\left(\frac{s_{(j)}}{r_{(j)}}\right)^{\frac{1}{2}}-1\right]\right\}^{\frac{1}{2}} \\
& \widetilde{\beta}_{(j)}=\left(\alpha_{(j)} \beta_{(j)}\right)^{\frac{1}{2}} .
\end{aligned}
$$

Isso posto, os estimadores corrigidos por viés, via jackknife, são dados por

$$
\begin{aligned}
& \bar{\alpha}=n \widetilde{\alpha}-(n-1) \widetilde{\alpha}, \\
& \bar{\beta}=n \widetilde{\beta}-(n-1) \widetilde{\beta},
\end{aligned}
$$

em que

$$
\widetilde{\alpha}=\frac{1}{n} \sum_{j=1}^{n} \widetilde{\alpha}_{(j)} \quad \text { e } \quad \widetilde{\beta}=\frac{1}{n} \sum_{j=1}^{n} \widetilde{\beta}_{(j)} .
$$

\subsection{Estimador do Método dos Momentos Modificados, corrigido por viés via Bootstrap $\stackrel{*}{\alpha}$ e $\stackrel{*}{\beta}$}

O método bootstrap, introduzido Bradley Efron [4], foi inspirado em uma metodologia baseada em reamostragem denominada jackknife. [4] sintetizou as metodologias baseadas em reamostragem que até então existiam e estabeleceu uma nova área de pesquisa.

Inicialmente houve ceticismo sobre a metodologia bootstrap, tendo sido tal ceticismo superado à medida em que estudos acumularam evidências de que o bootstrap pode ser consideravelmente mais eficaz que metodologias tradicionais.

A ideia de substituir aproximações complicadas e muitas vezes imprecisas por métodos de simulação baseados em reamostragem tem atraído diversos pesquisadores a desenvolver metodologias baseadas em bootstrap para os mais variados fins. Com a popularização do método bootstrap, alguns pesquisadores começaram a estabelecer condições matemáticas sob as quais o bootstrap é justificável.

Na literatura existem muitos trabalhos que fazem uso de metodologias bootstrap. Em geral, o método bootstrap é utilizado para correção de viés de estimadores, construção de intervalos de confiança, testes de hipóteses, estimação do erro-padrão de um estimador, entre outros.

As metodologias bootstrap apresentam dois enfoques, sendo eles o bootstrap paramétrico e o bootstrap não-paramétrico. Bootstrap paramétrico refere-se ao caso em que a reamostragem é feita com base em uma distribuição conhecida ou estabelecida. Em contrapartida, no bootstrap não-paramétrico há o desconhecimento da distribuição verdadeira $(F)$ dos dados. A reamostragem é feita com base na função de distribuição empírica $\widehat{F}_{n}$. Reamostrar de $\widehat{F}_{n}$ equivale a reamostar dos dados com reposição. 
Neste artigo, entretanto, o foco será na aplicação do bootstrap não-paramétrico para a correção do viés dos EMMMs. Em outras palavras, com o uso do bootstrap pode-se estimar o viés dos EMMMs. Isso é feito estimando-se $\mathbb{E}(\widetilde{\alpha})$ e $\mathbb{E}(\widetilde{\beta})$ a partir das respectivas médias dos estimadores obtidos nas $B$ réplicas bootstrap geradas. Tem-se então que

$$
\alpha^{*}=\frac{1}{B} \sum_{j=1}^{B} \alpha_{j}^{*} \quad \text { e } \quad \beta^{*}=\frac{1}{B} \sum_{j=1}^{B} \beta_{j}^{*} .
$$

Logo, a partir da definição de viés de um estimador, obtém-se as expressões dos EMMMs corrigidos por viés, via bootstrap, para os parâmetros $\alpha$ e $\beta$ como sendo

$$
\stackrel{*}{\alpha}=2 \widetilde{\alpha}-\alpha^{*} \text { e } \stackrel{*}{\beta}=2 \widetilde{\beta}-\beta^{*} .
$$

\section{RESULTADOS}

A fim de avaliar os desempenhos dos EMMMs e de suas versões corrigidas por viés, foram realizadas simulações de Monte Carlo na linguagem de programação C. Considerou-se o tamanho da amostra como $n=10,50,100$ e para o parâmetro $\alpha$ os valores 0,$25 ; 0,50 ; 1,00$, com o parâmetro $\beta$ mantido fixo em 1, 0. Utilizou-se 3000 réplicas de Monte Carlo, para cada estimador de $\alpha$ e $\beta$ considerado sendo que essas foram obtidas usando-se diversos tipos de processos de otimização disponíveis na linguagem C. Para os estimadores obtidos através do esquema bootstrap, foram consideradas 500 réplicas para cada um dos parâmetros. Os desempenhos dos estimadores considerados neste estudo foram avaliado em função das estatísticas: estimativa média e erro, este último sendo definido como a raiz quadrada do erro quadrático médio dos estimadores considerados. Os resultados obtidos para os EMMMs e suas versões corrigidas por viés constam nas Tabelas 1 e 2 .

Por meio da Tabela 1, percebe-se que as estimativas médias obtidas pelos estimadores corrigidos por jackknife e por bootstrap obtiveram desempenho bem semelhantes, ficando bem próximos dos verdadeiros valores dos parâmetros. Em especial, os estimadores $\left(\alpha^{*}, \beta^{*}\right)$ obtiveram melhor desempenho para $n=100$. Vale destacar ainda o fato dos EMMMs terem fornecido melhores estimativas medias em detrimento de sua versão corrigida por viés, tanto para o parâmetro $\alpha$, quanto para $\beta$; tal fato foi verificado para todos os tamanhos de amostras, como também para todos os valores de $\alpha$.

No que diz respeito ao erro, os EMMMs corrigidos por bootstrap apresentaram erros maiores ao estimar o parâmetro $\beta$ porém, para $n=50$ e $n=100$ esse estimador obteve desempenho bem próximo aos EMMMs corrigidos por jackknife. Em relação ao parâmetro $\alpha$, as estimativas fornecidas pelo esquema bootstrap obtiveram os menores erros para todos os tamanhos amostrais e para todos os valores de $\alpha$ considerados quando não considera-se os EMMMs. Análogo ao ocorrido com as estimativas de $\beta$, os EMMMs corrigidos por bootstrap e os EMMMs corrigidos por jackknife apresentaram desempenhos bastante próximos. Chamou atenção, entretanto que, para todos os cenários amostrais considerados e para todos os valores de $\alpha$, os EMMMs apresentaram desempenho superior, em detrimento de todas as versões corrigidas. 
Tabela 1: Avaliação de estimadores dos parâmetros da distribuição Birnbaum-Saunders para diferentes valores de $\alpha$ e $\beta=1,0$.

\begin{tabular}{cccccccccc}
\hline & & \multicolumn{4}{c}{ Estimador de $\alpha$} \\
\cline { 3 - 9 }$n$ & $\alpha$ & $\widetilde{\alpha}$ & $\check{\alpha}$ & $\bar{\alpha}$ & $\stackrel{*}{\alpha}$ & $\widetilde{\beta}$ & $\check{\beta}$ & $\bar{\beta}$ & ${ }^{*}$ \\
\hline \multirow{2}{*}{10} & 0,25 & 0,229 & 0,255 & 0,250 & 0,246 & 1,002 & 0,998 & 0,999 & 0,999 \\
& 0,50 & 0,458 & 0,509 & 0,499 & 0,493 & 1,010 & 0,993 & 0,998 & 1,000 \\
& 1,00 & 0,912 & 1,013 & 0,999 & 0,983 & 1,038 & 0,975 & 0,995 & 1,002 \\
\hline \multirow{2}{*}{50} & 0,25 & 0,246 & 0,251 & 0,250 & 0,250 & 0,999 & 0,999 & 0,998 & 0,998 \\
& 0,50 & 0,492 & 0,502 & 0,500 & 0,500 & 0,999 & 0,998 & 0,997 & 0,997 \\
& 1,00 & 0,983 & 1,003 & 1,000 & 0,999 & 1,003 & 0,995 & 0,995 & 0,995 \\
\hline \multirow{2}{*}{100} & 0,25 & 0,248 & 0,250 & 0,250 & 0,250 & 0,999 & 0,999 & 0,998 & 0,998 \\
& 0,50 & 0,496 & 0,501 & 0,500 & 0,500 & 0,998 & 0,999 & 0,997 & 0,997 \\
& 1,00 & 0,991 & 1,001 & 1,000 & 0,999 & 0,999 & 0,997 & 0,995 & 0,995 \\
\hline
\end{tabular}

1 - Médias das estimativas obtidas em 3 mil réplicas de Monte Carlo.

Tabela 2: Erros dos estimadores dos parâmetros da distribuição Birnbaum-Sauders para diferentes valores de $\alpha$ e $\beta=1,0$.

\begin{tabular}{cccccccccc}
\hline & & \multicolumn{4}{c}{ Estimador de $\alpha$} & \multicolumn{5}{c}{ Estimador de $\beta$} \\
\cline { 3 - 9 }$n$ & $\alpha$ & $\widetilde{\alpha}$ & $\check{\alpha}$ & $\bar{\alpha}$ & $\stackrel{*}{\alpha}$ & $\widetilde{\beta}$ & $\check{\beta}$ & $\bar{\beta}$ & $\stackrel{*}{\beta}$ \\
\hline \multirow{2}{*}{10} & 0,25 & 0,058 & 0,061 & 0,060 & 0,059 & 0,078 & 0,001 & 0,078 & 0,078 \\
& 0,50 & 0,117 & 0,123 & 0,121 & 0,119 & 0,155 & 0,006 & 0,153 & 0,153 \\
& 1,00 & 0,237 & 0,245 & 0,246 & 0,240 & 0,300 & 0,024 & 0,287 & 0,288 \\
\hline \multirow{2}{*}{50} & 0,25 & 0,025 & 0,025 & 0,025 & 0,025 & 0,034 & 0,000 & 0,034 & 0,034 \\
& 0,50 & 0,050 & 0,050 & 0,050 & 0,050 & 0,067 & 0,001 & 0,067 & 0,067 \\
& 1,00 & 0,100 & 0,100 & 0,100 & 0,100 & 0,124 & 0,005 & 0,123 & 0,123 \\
\hline \multirow{2}{*}{100} & 0,25 & 0,017 & 0,018 & 0,018 & 0,018 & 0,024 & 0,000 & 0,024 & 0,024 \\
& 0,50 & 0,035 & 0,036 & 0,036 & 0,036 & 0,047 & 0,000 & 0,047 & 0,047 \\
& 1,00 & 0,071 & 0,072 & 0,072 & 0,072 & 0,087 & 0,002 & 0,086 & 0,087 \\
\hline
\end{tabular}




\title{
5 CONCLUSÃO
}

Foi possível observar que os EMMMs corrigidos por bootstrap obtiveram, de maneira geral, desempenho superior em relaçao a todos os demais estimadores, porém, apresentaram os maiores erros, possivelmente por possuírem variâncias inflacionadas. Verificou-se também que os EMMMs corrigidos por viés propostos por [8] obtiveram, de maneira geral, desempenho inferior, quando comparados aos outros estimadores analisados. Menciona-se ainda que os EMMMs corrigidos por jackknife e os corrigidos por bootstrap apresentaram desempenho muito semelhantes, quando os tamanhos amostrais considerados foram $n=50$ e $n=100$, com uma leve vantagem para os estimadores obtidos pelo esquema bootstrap.

Em síntese, pode-se concluir, portanto, que os EMMMs corrigidos por viés, via bootstrap, são os mais recomendados para estimar os parâmetros $\alpha$ e $\beta$ da distribuição Birnbaum-Sauders em pequenas amostras e que, para tamanhos amostrais superiores a 50, é preferível o uso dos EMMMs corrigidos por jackknife, pois apresentaram erros (variâncias) menores que os EMMMs corrigidos por bootstrap. Os resultados obtidos nesse estudo estão limitados aos cenários considerados nas simulações apresentadas.

\begin{abstract}
This article aimed to evaluate the performances of the estimators of the Modified Method of Moments for the parameters $\alpha$ and $\beta$ Birnbaum-Saunders and three corrected versions of these estimators: the corrected version due to the bias, the bias corrected by means jackknife and corrected version due to the bias through bootstrap. Monte Carlo simulations were used to achieve the proposed objective, through the verification of some properties of these estimators, namely mean and error.
\end{abstract}

Keywords: Birnbaum-Saunders distribution, bias correction, jackknife and bootstrap estimates.

\section{REFERÊNCIAS}

[1] C.A.G. Araújo Júnior. "Ajustes para a verossimilança perfilada na distribuição Birnbaum-Saunders". 2006. f. 89. Dissertação (Mestrado em Estatística) - Departamento de Estatística - Universidade Ferderal de Pernambuco, Recife, (2006).

[2] Z.W. Birnbaum \& S.C. Saunders. A new family of life distribuitions. Journal of Applied Probability, 6 (1969), 319-327.

[3] D.J. Dupuis \& J.E. Mills. Robust estimation of the Birnbaum-Saunders distribution. IEEE Trans. Reliability, 47 (1998), 88-95.

[4] B. Efron \& R. Tibshiriani. An introduction to the bootstrap, Chapman and Hall, New york \& London, (1993).

[5] A.J. Lemonte. "Inferência Sobre Os Parâmetros da Distribuição Birnbaum-Saunders Bi-Paramétrica". 2006. f. 191. Dissertação (Mestrado em Estatística) - Departamento de Estatística, Universidade Ferderal de Pernambuco, Recife, (2006). 
[6] A.J. Lemonte, F.C. Cribari-Neto \& K.L.P. Vasconcellos. Improved statistical inference for the two parameter Birnbaum-Saunders distribution. Comput. Statist. Data Anal., 51 (2007), 4656-4681.

[7] P.S. Nascimento. Estimação de Máxima Verossimilhança dos Parâmetros da distribuição BirnbaumSaunders Utilizando a técnica de Otimização Não-Linear BFGS, Encontro Regional de Matemática Aplicada e Computacional, Natal, (2008).

[8] H.K.T. Ng, D. Kundu \& N. Balakrishan. Modified moment estimation for the two-parameter Birnbaum Saunders distribution. Comput. Statist. Data Anal., 43 (2003), 283-298. 